Comments, corrections, and related references welcomed, as always!

\title{
Can one factor the classical adjoint of a generic matrix?*
}

\author{
George M. Bergman
}

November 4, 2018

\begin{abstract}
Let $k$ be a field, $n$ a positive integer, $X$ a generic $n \times n$ matrix over $k$, i.e., a matrix $\left(x_{i j}\right)$ of $n^{2}$ independent indeterminates over the polynomial $\operatorname{ring} k\left[x_{i j}\right]$, and $\operatorname{adj}(X)$ its classical adjoint. It is shown that if char $k=0$ and $n$ is odd, then $\operatorname{adj}(X)$ is not the product of two noninvertible $n \times n$ matrices over $k\left[x_{i j}\right]$. I do not know whether the same is true in general; for char $k=0$ and $n$ even the possibilities are very limited.

The operation adj on matrices arises from the $(n-1)$ st exterior power functor on modules; the same question can be posed for matrix operations arising from other functors.
\end{abstract}

\section{Introduction}

If $A$ is an $n \times n$ matrix over a commutative ring $k$, and $\operatorname{adj}(A)$ its classical adjoint, i.e., the $n \times n$ matrix of appropriately signed minors of $A$, then

$$
\operatorname{det}(A) I_{n}=A \operatorname{adj}(A)
$$

[1] p.197, (5)], 4 Prop. XIII.4.16]. It is natural to ask whether the factors on the right above have any further factorizations.

To make this question precise, let $k\left[x_{i j}\right]$ be a polynomial ring in $n^{2}$ indeterminates $x_{i j}(1 \leq i, j \leq n)$. The matrix $X=\left(x_{i j}\right)$ is called a generic $n \times n$ matrix over $k$, and we may ask whether one can further refine the factorization

$$
\operatorname{det}(X) I_{n}=X \operatorname{adj}(X)
$$

as a factorization of $\operatorname{det}(X) I_{n}$ into noninvertible $n \times n$ matrices over $k\left[x_{i j}\right]$.

Note that the determinant of $\operatorname{det}(X) I_{n}$ is $\operatorname{det}(X)^{n}$. It follows, in view of (2), that $\operatorname{det}(\operatorname{adj}(X))=$ $\operatorname{det}(X)^{n-1}$. Moreover, for $k$ an integral domain, $\operatorname{det}(X)$ is irreducible over $k\left[x_{i j}\right]$. Indeed, $\operatorname{det}(X)$ is homogeneous of degree 1 in the entries of each row of $X$, hence any factor must be homogeneous of degree 1 or 0 in those entries; hence if one factor involves an $x$ from some row, no other factor can involve any $x$ from that row, hence that factor must involve all the $x$ 's from that row; moreover the same applies to columns. It follows that in any factorization, one factor must involve all the indeterminates and the other factors none; hence the others will belong to $k$, and indeed must be units of $k$, since the coefficients of the monomials in $\operatorname{det}(X)$ are \pm 1 .

It follows that in (2), $X$ cannot be factored further into noninvertible square matrices, and that any such factorization of the other term,

$$
\operatorname{adj}(X)=Y Z
$$

must, up to units of $k$, satisfy

$$
\operatorname{det}(Y)=\operatorname{det}(X)^{i}, \quad \operatorname{det}(Z)=\operatorname{det}(X)^{n-1-i}, \quad \text { where } 0<i<n-1 .
$$

*2000 Mathematics Subject Classifications. Primary: 15A23. Secondary: 12D05, 13A18, 14F05, 15A15, 55R25. E-mail address of author: gbergman@math.berkeley.edu. 
(This is clear if $k$ is a field because $k\left[x_{i j}\right]$ is a UFD. The case of a general integral domain may be obtained by applying this observation over the field of fractions of $k$, and again looking at monomials which have coefficient 1 in $\operatorname{det}(X)^{n}$.)

Given any ring homomorphism $\varphi$, let us also use the symbol $\varphi$ for the induced map on $n \times n$ matrices. Note that for each $n \times n$ matrix $A$ over a $k$-algebra $R$, there is a unique $k$-algebra homomorphism $\varphi_{A}$ : $k\left[x_{i j}\right] \rightarrow R$ carrying the generic matrix $X$ to $A$. This map $\varphi_{A}$ will therefore carry a factorization (3), if one exists, to a factorization of $\operatorname{adj}(A)$; and the entries of the factor matrices $\varphi_{A}(Y)$ and $\varphi_{A}(Z)$ will be given by polynomials in the entries of $A$. Hence if $R=k=$ the field of real or complex numbers, $\varphi_{A}(Y)$ and $\varphi_{A}(Z)$ will be continuous as functions of $A$. We shall get our main result by combining this observation with topological results from [2] and [3]. For $k$ of positive characteristic, it seems likely that algebraic geometry could take the place of topology, but to my knowledge the results we would need are not in the literature.

One caveat: If we had a factorization (3), the induced factorizations $\operatorname{adj}(A)=\varphi_{A}(Y) \varphi_{A}(Z)$ would be functorial, in the sense that they would respect homomorphisms among $k$-algebras, but it cannot be assumed that they would have other reasonable functoriality-like properties, even when these hold for adj itself. For instance, because the construction adj is induced by dualization (matrix transpose) followed by the $(n-1)$ st exterior power functor on modules, it satisfies the multiplicative relation

$$
\operatorname{adj}(A B)=\operatorname{adj}(B) \operatorname{adj}(A),
$$

but it does not follow that for $Y$ as in (3) we would have $\varphi_{A B}(Y)=\varphi_{B}(Y) \varphi_{A}(Y)$. Applying (11) to a matrix $U \in \mathrm{SL}_{n}(k)$, we get $\operatorname{adj}(U)=U^{-1}$, whence (5) yields $\operatorname{adj}\left(U A U^{-1}\right)=U \operatorname{adj}(A) U^{-1}$; but again, no such property can be assumed for $\varphi_{A}(Y)$.

On the other hand, let us note some valid consequences of functoriality in $k$. If we had a factorization (3) satisfying (4) over a base ring $k$, we would immediately get such a factorization over any ring to which $k$ can be mapped homomorphically; hence in proving nonexistence results for factorizations, results for algebraically closed fields $k$ will imply results for general $k$. Moreover, since a factorization of $\operatorname{adj}(X)$ over a given $k$ involves only finitely many elements of $k$, and any finitely generated field of characteristic 0 embeds in $\mathbb{C}$, nonexistence results with $k=\mathbb{C}$ will imply the corresponding results for all fields of characteristic 0 , and hence for all commutative rings of characteristic 0 .

\section{Valuations and ranks}

We shall show below, for $k$ a field, that if there exists a factorization (3), then, for appropriate families of matrices $A$, the matrices $\varphi_{A}(Y)$ will have constant rank. Varying $A$, we will get maps among Grassmannian varieties; it is to these that we will apply topological results in the next section. The constant-rank result will be proved using

Lemma 1. Let $R$ be a discrete valuation ring, with valuation $v$, maximal ideal $\mathbf{m}$, and residue map $\pi: R \rightarrow R / \mathbf{m}$. If $M$ is a square matrix over $R$ such that $\pi(M)$ has nullity $r$ (i.e., rank $n-r)$, then $v(\operatorname{det}(M)) \geq r$.

Proof. Left multiplication by some invertible matrix $\pi(U)$ over $R / \mathbf{m}$ turns $\pi(M)$ into a matrix whose last $r$ rows are zero. Since $\pi(U)$ is invertible, $v(\operatorname{det}(U))=0$, so $v(\operatorname{det}(M))=v(\operatorname{det}(U M))$, which is $\geq r$ since $U M$ has $r$ rows in $\mathbf{m}$.

Corollary 2. Let $p$ be an irreducible element in a unique factorization domain $R$, $v_{p}$ the corresponding valuation on $R$, and $\pi_{p}: R \rightarrow R / p R$ the residue map. Then for $M$ a square matrix over $R, \pi_{p}(M)$ has rank at least $n-v_{r}(\operatorname{det}(M))$.

Proof. Localize at $p R$, and apply the preceding lemma in contrapositive form.

Using the above results we can now prove

Lemma 3. Let $X=\left(x_{i j}\right)$ be a generic $n \times n$ matrix over a field $k$, and suppose $\operatorname{adj}(X)$ admits a factorization (3) satisfying (4) for some $i$. Let $A$ be any $n \times n$ matrix over $k$ which has the eigenvalue 0 with multiplicity exactly 1 , and let $\varphi_{A}$ denote the homomorphism $k\left[x_{i j}\right] \rightarrow k$ taking $X$ to $A$. Then

$$
\operatorname{rank}\left(\varphi_{A}(Y)\right)=n-i, \quad \operatorname{rank}\left(\varphi_{A}(Z)\right)=i+1, \quad \operatorname{rank}\left(\varphi_{A}(X Y)\right)=n-1-i, \quad \operatorname{rank}\left(\varphi_{A}(Z X)\right)=i .
$$


Proof. Let $k[t]$ be a polynomial ring in one indeterminate, and $v_{t}$ the valuation on this ring induced by the element $t$. From the hypothesis on $A$, we see that $\operatorname{det}\left(t I_{n}+A\right)$, i.e., the characteristic polynomial of $-A$, has constant term 0 but nonzero coefficient of $t$, so $v_{t}\left(\operatorname{det}\left(t I_{n}+A\right)\right)=1$. Writing $\psi: k\left[x_{i j}\right] \rightarrow k[t]$ for $\varphi_{\left(t I_{n}+A\right)}$, i.e., the $k$-algebra homomorphism taking $X$ to $t I_{n}+A$, we get

$$
v_{t}(\operatorname{det}(\psi(Y)))=v_{t}(\psi(\operatorname{det}(Y)))=v_{t}\left(\psi\left(\operatorname{det}(X)^{i}\right)\right)=v_{t}\left(\operatorname{det}\left(t I_{n}+A\right)^{i}\right)=i .
$$

Letting $\pi_{t}: k[t] \rightarrow k$ take $t$ to 0 , we have $\pi_{t} \psi=\varphi_{A}$, hence applying Corollary [2] to (6) we get $\operatorname{rank}\left(\varphi_{A}(Y)\right) \geq n-i$. Similarly, $\operatorname{rank}\left(\varphi_{A}(Z)\right) \geq n-(n-1-i)=i+1$.

On the other hand, note that $A \varphi_{A}(Y) \varphi_{A}(Z)=\varphi_{A}(X Y Z)=\varphi_{A}\left(\operatorname{det}(X) I_{n}\right)=\operatorname{det}(A) I_{n}=0$, so the nullities of $A$, of $\varphi_{A}(Y)$ and of $\varphi_{A}(Z)$ must add up to at least $n$, i.e., their ranks can sum to at most $2 n$. Since the rank of the first is $n-1$ and those of the other two are at least $n-i$ and $i+1$, these must be their exact values. The final two equalities are seen similarly. (In obtaining the last one, we use (2) in the form $\operatorname{det}(X) I_{n}=\operatorname{adj}(X) X$, easily deduced from the form given.)

Remark: The above hypothesis that the the eigenvalue 0 have multiplicity 1 is stronger than saying that $A$ has rank $n-1$. For example, the matrix consisting of a single $n \times n$ Jordan block with eigenvalue 0 has rank $n-1$, but that eigenvalue has multiplicity $n$.

We will formulate our next result in algebraic-geometric terms. For base field $\mathbb{R}$ or $\mathbb{C}$, this formulation implies the corresponding topological statement, which is what we will actually use in the next section; but the algebraic-geometric statement has the potential of yielding results in positive characteristic as well.

For $0 \leq i \leq n$, let $\operatorname{Gr}_{k}(i, n)$ denote the Grassmannian variety over $k$ whose $K$-valued points, for a field $K$ over $k$, correspond to $i$-dimensional subspaces $V_{i} \subseteq K^{n}$. On the other hand, let $\operatorname{CGr}_{k}(i, n)$ (for "complemented Grassmannian") denote the variety whose $K$-valued points correspond to pairs $\left(V_{i}, V_{n-i}\right)$ consisting of an $i$-dimensional subspace $V_{i}$ and an $(n-i)$-dimensional subspace $V_{n-i}$ such that $K^{n}=$ $V_{i} \oplus V_{n-i}$.

The variety $\operatorname{Gr}_{k}(i, n)$ is projective; in particular $\operatorname{Gr}_{k}(1, n)$ is $(n-1)$-dimensional projective space. On the other hand, $\operatorname{CGr}_{k}(i, n)$ is affine, since it can be identified with the variety of idempotent $n \times n$ matrices of rank $i$.

Proposition 4. Suppose $\operatorname{adj}(X)$ admits a factorization (3) satisfying (4) for some $i$. Then there exists a morphism of varieties $\operatorname{CGr}_{k}(1, n) \rightarrow \operatorname{Gr}_{k}(i, n)$ which takes every pair $\left(V_{1}, V_{n-1}\right)$ to a subspace of its second component $V_{n-1}$, and likewise there exists a morphism $\operatorname{CGr}_{k}(1, n) \rightarrow \operatorname{Gr}_{k}(n-1-i, n)$ with the same property.

Proof. Given a $K$-valued point $a=\left(V_{1}, V_{n-1}\right)$ of $\operatorname{CGr}_{k}(1, n)$, let $E_{a}$ denote the idempotent matrix over $K$ that projects $K^{n}$ onto $V_{n-1}$ along $V_{1}$. This has eigenvalue 0 with multiplicity 1 , hence by Lemma 3 . $E_{a} \varphi_{E_{a}}(Y)$ has rank $n-1-i$, so its column space is a subspace of $V_{n-1}$ of that rank. This construction can be seen to give a morphism of varieties, the second of the maps referred to.

To get the first, note that taking the transpose of the equation (3i) and applying it to the transpose of the matrix $X$, we get a factorization $\operatorname{adj}(X)=Z^{\prime} Y^{\prime}$ with $\operatorname{det}\left(Y^{\prime}\right)=\operatorname{det}(Y)$ and $\operatorname{det}\left(Z^{\prime}\right)=\operatorname{det}(Z) . \operatorname{Applying}$ the preceding result to this factorization gives the desired morphism.

\section{The hairy sphere raises its unkempt head.}

For $n \leq 2$, the condition $0<i<n-1$ of (4) cannot be satisfied, so the first case where a factorization (3) is possible is when $n=3, i=1$. Suppose we had such a factorization for $k=\mathbb{R}$. Every point $p$ of the unit sphere $S^{2}$ determines a point $\left(\mathbb{R} p,(\mathbb{R} p)^{\perp}\right)$ of $\mathrm{CGr}_{\mathbb{R}}(1,3)$, so composing with the first morphism of Proposition 4 we would have a continuous map $S^{2} \rightarrow \operatorname{Gr}_{\mathbb{R}}(1,3)$ taking each $p \in S^{2}$ to a 1-dimensional subspace of $(\mathbb{R} p)^{\perp}$; in other words, of the tangent space to $S^{2}$ at $p$. This would constitute a "combing of a hairy sphere", which is known to be impossible. (The "hairy sphere" result as generally formulated concerns a choice of an oriented tangent direction at each point, while the above construction would give an unoriented direction. However, such an unoriented combing, if it existed, could be regarded as a map from $S^{2}$ to projective 2-space, and the simple connectedness of $S^{2}$ would allow us to lift it to a map to the universal covering space thereof, $S^{2}$, yielding an oriented combing.) 
For higher $n$, we can use in place of the hairy sphere theorem the results of [2] or [3]. As with $\mathrm{CGr}_{\mathbb{R}}(1,3)$ and $\operatorname{Gr}_{\mathbb{R}}(1,3)$ in the preceding paragraph, in the proof of the next theorem we shall regard varieties $\operatorname{Gr}_{\mathbb{C}}(i, n)$ and $\operatorname{CGr}_{\mathbb{C}}(i, n)$ as topological manifolds, so that "point of" and "continuous map" will make sense.

Theorem 5. Suppose char $k=0$. Then if $n$ is odd, there is no factorization (3) of $\operatorname{adj}(X)$ into noninvertible matrices, while if $n$ is even, such a factorization, if it exists, must have one of the exponents in (4) equal to 1 , i.e., must have $i=1$ or $n-2$.

Proof. As noted in the last paragraph of $₫ 1$ we may assume without loss of generality that $k=\mathbb{C}$. If we put a Hermitian inner product on $\mathbb{C}^{n}$, then the map $L \mapsto\left(L, L^{\perp}\right)$ takes $\operatorname{Gr}_{\mathbb{C}}(1, n)$ to $\operatorname{CGr}_{\mathbb{C}}(1, n)$. Now if we have a factorization (3), Proposition 4 gives us a continuous map $\operatorname{CGr}_{\mathbb{C}}(1, n) \rightarrow \operatorname{Gr}_{\mathbb{C}}(i, n)$ taking $\left(V_{1}, V_{n-1}\right)$ to a subspace of its second component. Composing, we get a continuous function taking each 1-dimensional subspace $L \subseteq \mathbb{C}^{n}$ to an $i$-dimensional subspace $L^{\prime} \subseteq L^{\perp}$.

This gives an $i$-dimensional subbundle of the tangent bundle on $n$-dimensional projective space, which by [3] Theorem 1.1(ii)] is possible only if $n$ is even and $i=1$ or $n-2$. Alternatively we may note that the map $L \mapsto L \oplus L^{\prime}$ ( $L^{\prime}$ as in the preceding paragraph) takes each 1-dimensional subspace $L$ of $\mathbb{C}^{n}$, to an $(i+1)$-dimensional subspace containing $L$, which by [2, Theorem 1.5(a)] can only happen if $n$ is even and $i+1=2$ or $n-1$, i.e., $i=1$ or $n-2$.

\section{The open cases}

Examples 1.2 and 1.3 of [2] show that for $n$ even there do exist continuous maps from $\operatorname{Gr}_{\mathbb{C}}(1, n)$ to $\operatorname{Gr}_{\mathbb{C}}(2, n)$ and to $\operatorname{Gr}_{\mathbb{C}}(n-1, n)$ taking each 1-dimensional subspace to a subspace containing it. (For a variant of the former example, give $\mathbb{C}^{n}$ a structure of $n / 2$-dimensional vector space over the division algebra $\mathbb{H}$ of quaternions, and take every 1-dimensional complex subspace $L$ to the 1-dimensional quaternionic subspace it spans.) I do not know whether for such $n$ there exist factorizations (3) such that the $i$ of (4) is 1 or $n-2$. (The existence of factorizations of these two sorts are equivalent; cf. end of proof of Proposition 4 )

I also do not know whether Theorem 5 remains true for fields $k$ of positive characteristic. The analog of [2. Theorem 1.5(a)] with morphisms of algebraic varieties over fields of arbitrary characteristic in place of continuous maps of topological spaces holds by [ibid., Theorem 1.5(b)]. In fact, for $n$ even, the latter result only allows an increasing map $\operatorname{Gr}_{k}(1, n) \rightarrow \operatorname{Gr}_{k}(i, n) \quad(1<i<n)$ when $i=n-1$, and not, as in the topological case, when $i=2$ as well. Unfortunately, the map $L \mapsto\left(L, L^{\perp}\right)$ which we used to connect Proposition 4 with [2, Theorem 1.5(a)] is not a morphism of algebraic varieties, and so cannot be used here. (It is based on a Hermetian inner product, which is not bilinear but sesquilinear; a genuine bilinear form on $\mathbb{C}^{n}$ cannot be positive definite. And if one retreats to the case $k=\mathbb{R}$ and tries to use a real inner product, this will not keep its positive definiteness at nonreal points, hence will not lead to a morphism of varieties.) Indeed, there can be no nontrivial morphism of algebraic varieties $\operatorname{Gr}_{k}(1, n) \rightarrow \mathrm{CGr}_{k}(1, n)$, since $\operatorname{Gr}_{k}(1, n)$ is projective while $\operatorname{CGr}_{k}(1, n)$ is affine.

The best hope for extending Theorem $[5$ to arbitrary characteristic seems to be to get some version of [2. Theorem 1.5(b)] for morphisms $\operatorname{CGr}_{k}(1, n) \rightarrow \operatorname{Gr}_{k}(i, n)$. If [2] Theorem 1.5(b)] generalizes in its entirety to such maps, this would not only extend our results to arbitrary characteristic, but also exclude the possible factorization for even $n$ allowed by Theorem [5 since such a factorization would lead to maps of the above sort for both $i=2$ and $i=n-1$, while [2, Theorem 1.5(b)] allows such maps only for $i=n-1$.

\section{$5 \quad$ Further questions}

In the context of Proposition [4 it is not clear what the relation between the two maps, $\operatorname{CGr}_{k}(1, n) \rightarrow$ $\operatorname{Gr}_{k}(i, n)$ and $\operatorname{CGr}_{k}(1, n) \rightarrow \operatorname{Gr}_{k}(n-1-i, n)$ arising from a factorization (3) would be. In view of the dimensions of the subspaces of $V_{n-1}$ that they yield, one can ask whether they would give a direct sum decomposition of that space.

Taking a more extravagant goal than we have done above, we may ask whether one can describe all maximal factorizations of the matrix $\operatorname{det}(X) I_{n}$ into noninvertible matrices over $k\left[x_{i j}\right]$. Aside from the factorization (2), the same factorization with the order of factors reversed, and the two factorizations arising similarly from the transpose of $X$, there is the factorization into $n$ diagonal matrices each having 
determinant $\operatorname{det}(X)$,

$$
\operatorname{det}(X) I_{n}=\operatorname{diag}(\operatorname{det}(X), 1, \ldots, 1) \cdot \operatorname{diag}(1, \operatorname{det}(X), 1, \ldots, 1) \cdot \ldots \cdot \operatorname{diag}(1, \ldots, 1, \operatorname{det}(X)) .
$$

Are (2), its three variants noted above, and (7), "essentially" all there are? A factorization can be trivially perturbed by multiplying any two adjacent factors on the right and left respectively by an invertible matrix over $k\left[x_{i j}\right]$ and its inverse. Also, because $\operatorname{det}(X) I_{n}$ is central, we can multiply the first factor in a factorization on the left by an invertible matrix $U$, and the last factor by $U^{-1}$ on the right. So we may ask whether the five factorizations we have described form a set of representatives of the orbits of maximal factorizations under such perturbations.

In those five factorizations, the degree of the product matrix $\operatorname{det}(X) I_{n}$ in the $n^{2}$ indeterminates is precisely the maximum of the sums of the degrees of the matrix entries that are multiplied together; i.e., there is no "cancellation" in the calculation of the leading terms of the highest-degree entries of $\operatorname{det}(X) I_{n}$. We can easily destroy this property by perturbing our factorizations as above, using invertible matrices $U$ over $k\left[x_{i j}\right]$ with entries of high degree. Is there, however, some principle saying that any factorization of a "good" matrix over a polynomial ring is a perturbation, in this sense, of a factorization in which the degree is well-behaved?

Finally, let us observe that for an $n \times n$ matrix $A$ over a commutative ring $k$, representing a linear map $a: k^{n} \rightarrow k^{n}$, the classical adjoint $\operatorname{adj}(A)$ can be characterized as the transpose of the matrix representing the linear map $\wedge^{n-1} a: \wedge^{n-1} k^{n} \rightarrow \wedge^{n-1} k^{n}$, where $\wedge^{n-1}$ denotes the $(n-1)$ st exterior power functor. If we apply a lower exterior power functor $\wedge^{m}$, we get an endomorphism of the module $\wedge^{m} k^{n}$, which is free of rank $\left(\begin{array}{c}n \\ m\end{array}\right)$. Again taking for $A$ a generic matrix $X$, we may ask whether the resulting $\left(\begin{array}{c}n \\ m\end{array}\right) \times\left(\begin{array}{l}n \\ m\end{array}\right)$ matrix over $k\left[x_{i j}\right]$ can be factored into noninvertible square matrices. (This matrix, incidentally, has determinant $\operatorname{det}(X)^{\left(\begin{array}{c}n-1 \\ m-1\end{array}\right)}$, and its product with the transpose of the matrix representing $\wedge^{n-m} a$, with rows and columns appropriately indexed, is $\operatorname{det}(X)$ times the $\left(\begin{array}{l}n \\ m\end{array}\right) \times\left(\begin{array}{l}n \\ m\end{array}\right)$ identity matrix.)

Each of the above functors $\wedge^{m}$ is a subfunctor of the $m$-fold tensor product functor $\otimes^{m}$; indeed, when char $k=0, \otimes^{m}$ decomposes into a direct sum of subfunctors indexed by Young diagrams. (The functor $\wedge^{m}$ corresponds to the height- $m$ column of boxes, while the $m$ th symmetric power functor corresponds to the length- $m$ row of boxes.) Thus, one may pose for each such functor the same question we have asked for $\wedge^{m}$.

\section{Acknowledgement}

I am indebted to Zinovy Reichstein for an extensive and valuable correspondence on this subject.

\section{References}

[1] P. M. Cohn, Algebra, second edition, v.1, Wiley \& Sons, 1982. MR 83e:00002.

[2] C. de Concini and Z. Reichstein, Nesting maps of Grassmannians, preprint Nov. 2002, 11 pp., readable at http://www.math.ubc.ca/ reichst/pub.html .

[3] H. Glover, W. Homer and R. Stong, Splitting the tangent bundle of projective space, Indiana University Math Journal, 31 (1982), 161-166. MR 83f:57016.

[4] Serge Lang, Algebra, Addison-Wesley, third edition, 1993, reprinted as Springer Graduate Text in Mathematics v.211, 2002. MR 2003e:00003. 